\title{
Multiple Criteria Quadratic Programming for Financial Distress Prediction of the Listed Manufacturing Companies
}

\author{
Ying Wang ${ }^{1}$, Peng Zhang ${ }^{1}$, Guangli Nie ${ }^{1}$, and Yong Shi ${ }^{1,2, *}$ \\ ${ }^{1}$ Research Center on Fictitious Economy \& Data Science, Chinese Academy of Sciences, \\ Beijing, 100190, China \\ ${ }^{2}$ College of Information Science \& Technology, University of Nebraska at Omaha, \\ Omaha, NE 68182, USA \\ wangying.bj.cn@gmail.com, nczhang1999@163.com, \\ sdungl@163.com, yshi@gucas.ac.cn
}

\begin{abstract}
Nowadays, how to effectively predict financial distress has become an important issue for companies, investors and many other user groups. The purpose of this paper is to apply the Multiple Criteria Quadratic Programming (MCQP) model to predict financial distress of the listed manufacturing companies. Firstly, we introduce the formulation of MCQP model. Then we use ten-folder cross validation to test the stability and accuracy of MCQP model on a real-life listed companies' financial ratios dataset. At last, we compare MCQP model with other two well-known models: Logistic Regression and SVM models. The experimental results show that MCQP is accurate and stable for predicting the financial distress of the listed manufacturing companies. Consequently, we can safely say that MCQP is capable of providing stable and credible results in predicting financial distress.
\end{abstract}

Keywords: Financial distress prediction, MCQP, Logistic, SVM.

\section{Introduction}

With the rapid development of capital market in recent years, making accurate financial distress prediction has become more and more important to many user groups, such as bank loan officers, investors, creditors, regulators and auditors [1]. The last few decades have witnessed a large body of research work on predicting finance distress from financial statement. As early as 1966, Beaver [2] discussed that the default prediction problem could be regarded as a problem of evaluating the probability of financial distress conditional upon the value of a specific financial ratio. In 1968, Altman [3] developed a Z-score bankruptcy prediction model based on five financial ratios using Multiple Discriminant Analysis (MDA). In 1980, Ohlson [4] applied the logistic regression model in bankruptcy prediction research. Unlike MDA, the logistic regression model does not based on normal distribution or the equality of covariance matrices of the two groups. He was followed by several other authors:

\footnotetext{
* Corresponding author.
} 
Mensah [5], Casey and Bartczak [6] and Gentry at al [7]. From the late 1980s, the Artificial Intelligence (AI) or Machine Learning (ML) techniques were introduced to financial distress prediction studies [8, 9]. Most recently, $\mathrm{Yu}-\mathrm{Chiang} \mathrm{Hu}$ and Jake Ansell [10] applied the SVM model which based on convex quadratic programming to predict financial distress.

In recent years, researchers from Multiple Criteria Mathematical Programming (MCMP) are stepping into data mining field and propose many promising classification models. For instance, in 2001, Y. Shi [11] built up the Multiple Criteria Linear Programming (MCLP) and Multiple Criteria Quadratic Programming (MCQP) models which have received many attentions as their successful applications in finance, biology, medical insurance and many other social fields. The purpose of this paper is to apply the MCQP model to predict the financial distress of the listed manufacturing companies in China.

The rest of this paper is organized as follows: in Section 2, we introduce the MCQP model; in Section 3, we introduce the financial ratios dataset of the listed manufacturing companies; in Section 4, we test MCQP on this dataset using 10-folder cross-validation and compare its performance with two well-known models: Logistic Regression and SVM; in Section 5, we conclude our paper with some discussions.

\section{Multiple Criteria Quadratic Programming (MCQP) Model}

In this section, we will give a short introduction of MCQP model. Assume a twogroup classification problem $\left\{G_{1}, G_{2}\right\}$. Given a training sample $T_{r}=\left\{G_{1}, G_{2}\right\}$, where $n$ is the total number of records in the training sample. Each training instance $A_{i}(i=1, \ldots n)$ has $r$ attributes. A boundary scalar $b$ is used to separate $G_{1}$ and $G_{2}$. Thus a vector $X=\left(x_{1}, x_{2} \ldots x_{n}\right) \in R^{r}$ can be identified to establish the following linear inequality [12]:

$$
\begin{aligned}
& A_{i} X<b, \text { some } A_{i} \in G_{1} \\
& A_{i} X \geq b, \text { some } A_{i} \in G_{2}
\end{aligned}
$$

To formulate the criteria and complete constraints for data separation, some variables will be introduced. $\alpha_{i}$ is defined to measure the overlapping of two-group boundary for record $A_{i}$, that means if $A_{i} \in G_{1}$ but we misclassified it into $G_{2}$ or vice versa, there is a distance $\alpha_{i}$ and the value equals $\left|A_{i} X-b\right|$. Then $\beta_{i}$ is defined to measure the distance of record $A_{i}$ from its adjusted boundary $b^{*}$, that means if $A_{i}$ is correctly classified, there is a distance $\beta_{i}$ and the value equals $\left|A_{i} X-b^{*}\right|$, where $b^{*}=b+\alpha_{i}$ or $b^{*}=b-\alpha_{i}$. Suppose $f(\alpha)$ denotes for the relationship of all overlapping $\alpha_{i}$ while $g(\beta)$ denotes for the aggregation of all distances $\beta_{i}$. The final absolute catch rates depend on simultaneously minimizing $f(\alpha)$ and maximizing $g(\beta)$. By using the $l_{p}$ norm to represent $f(\alpha)$ and $l_{q}$ norm to represent $g(\beta)$ respectively, we get a generalized bi-criteria programming framework as follows: 


$$
\begin{gathered}
\text { Minimize }\|\alpha\|_{p}^{p} \text { and maxmize }\|\beta\|_{q}^{q} \\
\text { subject to }: \\
\text { AiX }-\alpha_{i}+\beta_{i}-b=0, A_{i} \in G_{1} \\
A_{i} X+\alpha_{i}-\beta_{i}-b=0, A_{i} \in G_{2} \\
\alpha_{i}, \beta_{i} \geq 0, i=1, \ldots, n
\end{gathered}
$$

Where $A i$ is given, $X$ and $b$ are unrestricted.

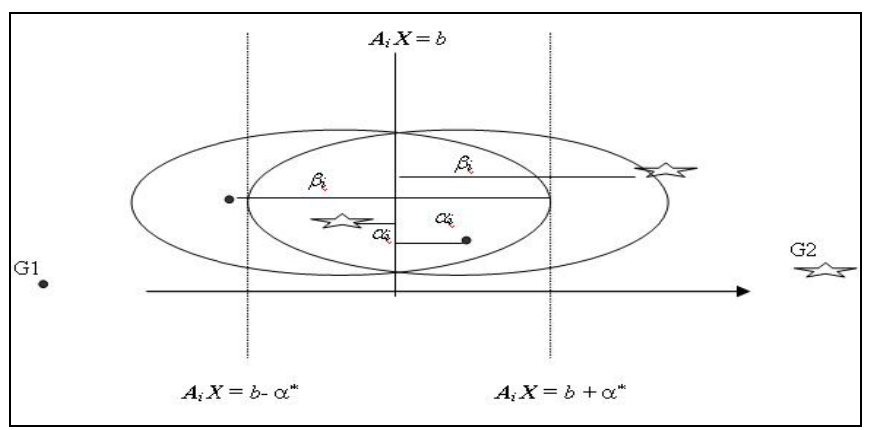

Fig. 1. Two-group classification model

All variables and their relationships are represented in Figure 1. There are two groups in Figure 1: black dot indicates $G_{1}$ data objects and star indicates $G_{2}$ data objects. There is one misclassified data object from each group if the boundary scalar $b$ is used to classify these two groups, whereas adjusted boundaries $b-\alpha_{i}$ and $b+\alpha_{i}$ separate two groups without misclassification.

As far as Model 1 is considered, when setting $\mathrm{p}=\mathrm{q}=2$ and combing the two objective functions into a single objective function by using $w_{\alpha} \geq 0$ and $w_{\beta} \geq 0$, we can get the MCQP model as follows:

$$
\begin{gathered}
\text { Minimize } w_{\alpha} \sum_{i=1}^{n} \alpha_{\mathrm{i}}^{2}-w_{\beta} \sum_{i=1}^{n} \beta_{i}^{2} \\
\text { subject to }: \\
A_{i} x-\alpha_{i}+\beta_{i}-b=0, \forall A_{i} \in G_{1} \\
A_{i} x+\alpha_{i}-\beta_{i}-b=0, \forall A_{i} \in G_{2} \\
\alpha_{i}, \beta_{i} \geq 0, i=1, \ldots, n
\end{gathered}
$$

Model 2 is a non-convex problem, thus it is also a NP-hard problem. It is very difficult to get the global minimizer, especially for large problem. In order to solve (3) efficiently, we propose an algorithm, which converges to a local minimizer of (2). 
In order to describe the algorithm in detail, we introduce some notation.

Let $\omega=(X, \alpha, \beta, b), \quad f_{1}(\omega)=w_{\alpha} \sum_{i=1}^{n} \alpha_{i}^{2}-w_{\beta} \sum_{i=1}^{n} \beta_{i}^{2}$, and $\Omega=\left\{\begin{array}{c}(X, \alpha, \beta, b): A_{i} X-\alpha_{i}+\beta_{i}-b=0, \forall i \in G_{1}, \\ A_{i} X+\alpha_{i}-\beta_{i}-b=0, \forall i \in G_{2}, \\ \alpha_{i} \geq 0, \beta_{i} \geq 0, i=1, \cdots, n\end{array}\right\}$ be the feasible region of model 2.

Let $\chi_{\Omega}(\omega)$ be the index function of set $\Omega$, i.e. $\chi_{\Omega}(\omega)$ is defined as follows

$$
\chi_{\Omega}(\omega)=\left\{\begin{array}{l}
0, \omega \in \Omega \\
+\infty, \omega \notin \Omega .
\end{array}\right.
$$

Then (3) is equivalent to the following problem

$$
\min f_{1}(\omega)+\chi_{\Omega}(\omega)
$$

Rewrite $f_{1}(\omega)+\chi_{\Omega}(\omega) \quad$ as $\quad f_{1}(\omega)+\chi_{\Omega}(\omega)=g_{1}(\omega)-h_{1}(\omega) \quad$. Where $g_{1}(\omega)=\frac{1}{2} \rho\|\omega\|^{2}+w_{\alpha} \sum_{i=1}^{n} \alpha_{i}^{2}+\chi_{\Omega}(\omega), h_{1}(\omega)=\frac{1}{2} \rho\|\omega\|^{2}+w_{\beta} \sum_{i=1}^{n} \beta_{i}^{2}, \rho>0$ is a small positive number. Then $g_{1}(\omega)$ and $h_{1}(\omega)$ are convex functions. Apply the simplified DC algorithm [13] to problem (4), we get the solution as follow:

Algorithm 1. Given initial point $\omega^{0} \in R^{3 n+1}$ and parameter $\varepsilon>0$ at each iteration $k \geq 1$, compute $\omega^{k+1}$ by solving the convex quadratic programming.

$\left(Q^{k}\right) \min \left\{\frac{1}{2} \rho\|\omega\|^{2}+\sum_{i=1}^{n} \alpha_{i}^{2}-\left(h_{1}{ }^{\prime}\left(\omega^{k}\right), \omega\right), \omega \in \Omega\right\}$

The stopping criterion is $\left\|\omega^{k+1}-\omega^{k}\right\| \leq \varepsilon$. The sequence $\left\{w^{k}\right\}$ generated by Algorithm 1 converges to a local minimizer of (3).

\section{Financial Ratios Dataset}

The sample is selected from the manufacturing companies listed in the Shanghai Stock Exchange (SSE) and the Shenzhen Stock Exchange (SZSE). Financial states of these companies are categorized into two classes: healthy and distressed. Companies which are Specially Treated (ST) by China Securities Supervision and Management Committee (CSSMC) will be taken as the distressed ones, while those which are never specially treated by CSSMC will be seen as the healthy ones. 775 manufacturing companies are selected, of which 46 are ST companies and the remained 729 are healthy ones. For healthy companies, their financial statements in 
the last year will be used, while for ST companies, their financial statements in the year before the special treated year ${ }^{1}$ will be used.

In this paper, we use the financial statements between 2003 and 2007, then 40 ST companies are selected out of the total 46 ST companies. Due to the scarcity of ST companies, a matched-pair design is used to compose the examples. Each ST company is matched with two healthy companies randomly selected from the 729 healthy companies. By doing so, the sample is composed of 120 listed manufacturing companies, including $40 \mathrm{ST}$ records and 80 healthy records.

According to the standards of summarization, measurability, and sensitivity, 24 financial rations which derived from the financial statements are calculated for the

Table 1. Descriptive statistics of the financial ratios $(\mathrm{N}=120)$

\begin{tabular}{|c|c|c|c|c|c|c|}
\hline Principle & $\begin{array}{l}\text { Vari- } \\
\text { ables }\end{array}$ & Financial ratios & Min. & Max. & Mean & StdDev \\
\hline \multirow[t]{4}{*}{ Liability } & 1 & Current ratio & 0.175 & 6.300 & 1.406 & 0.976 \\
\hline & 2 & Acid-test ratio & 0.000 & 5.534 & 0.995 & 0.779 \\
\hline & 3 & $\begin{array}{l}\text { Net cash flow from operating } \\
\text { activities / Current liability }\end{array}$ & -10.54 & 1.976 & 0.05 & 1.066 \\
\hline & 4 & Current liability / Total liability & -0.988 & 2.629 & 0.914 & 0.269 \\
\hline \multirow[t]{7}{*}{$\begin{array}{l}\text { Operational } \\
\text { Efficiency }\end{array}$} & 5 & $\begin{array}{l}\text { Turnover rate of } \\
\text { account receivable }\end{array}$ & 0.202 & 26.20 & 4.665 & 3.449 \\
\hline & 6 & Turnover rate of inventory & 0.243 & 4738 & 50.79 & 431.9 \\
\hline & 7 & Turnover rate of total assets & 0.019 & 5.49 & 0.917 & 0.719 \\
\hline & 8 & Cost of sales / Revenue & 0.056 & 1.116 & 0.745 & 0.207 \\
\hline & 9 & $\begin{array}{l}\text { Selling \& distribution expense / } \\
\text { Revenue }\end{array}$ & 0.003 & 0.938 & 0.075 & 0.107 \\
\hline & 10 & G\&A expence /Revenue & 0.005 & 27.14 & 0.424 & 2.484 \\
\hline & 11 & Finance expence / Revenue & -0.006 & 13.93 & 0.166 & 1.273 \\
\hline \multirow[t]{3}{*}{ Profitability } & 12 & Asset Profit Ratio & -1.666 & 0.475 & 0.029 & 0.289 \\
\hline & 13 & Return on assets & -1.683 & 0.462 & 0.009 & 0.277 \\
\hline & 14 & Net profit margin & -28.66 & 0.391 & -0.538 & 3.338 \\
\hline \multirow[t]{5}{*}{ Growth } & 15 & Revenue growth ratio & -0.778 & 2.993 & 0.226 & 0.466 \\
\hline & 16 & Net profit growth ratio & -523.5 & 11.47 & -11.50 & 56.84 \\
\hline & 17 & Total assets growth ratio & -0.703 & 3.542 & 0.248 & 0.496 \\
\hline & 18 & Equity growth ratio & -19.53 & 3.829 & 0.068 & 2.455 \\
\hline & 19 & Gross profit growth ratio & -1.305 & 3.716 & 0.18 & 0.733 \\
\hline \multirow[t]{3}{*}{ Structure } & 20 & Asset-liability ratio & 0.114 & 3.092 & 0.598 & 0.372 \\
\hline & 21 & Long-term liabilities /Total assets & -0.080 & 0.813 & 0.066 & 0.108 \\
\hline & 22 & $\begin{array}{l}\text { Fixed asset, other assets \& } \\
\text { intangible assets / Total assets }\end{array}$ & 0.000 & 0.798 & 0.387 & 0.167 \\
\hline \multirow[t]{2}{*}{ Cash flow } & 23 & $\begin{array}{l}\text { Cash received from sales of goods } \\
\text { or rendering services / Revenue }\end{array}$ & 0.561 & 5.846 & 1.085 & 0.479 \\
\hline & 24 & $\begin{array}{l}\text { Net cash flow from operating } \\
\text { activities / Net profit }\end{array}$ & -3.871 & 10.81 & 0.652 & 1.295 \\
\hline
\end{tabular}

${ }^{1}$ The Year before the Special Treated Year (YSTY) is defined as follows: suppose the time when a company is specially treated as the benchmark year $t_{0}$. If the company is specially treated among January to April, the YSTY is defined as two years before $t_{0}$. If the company is specially treated among May to December, YSTY is defined as the last year before $t_{0}$. 
120 companies. These financial rations cover liability ratios, operational efficiency ratios, profitability ratios, growth ratios, structure ratios and cash flow ratios which are listed in Table 1.

\section{Experiments}

\subsection{Empirical Study}

Cross-validation is frequently used for estimating generalization error, model selection, experimental design evaluation, training exemplars selection, or pruning outliers. By definition, cross-validation is the practice of partitioning a sample of data into sub samples so that analysis is initially performed on a single sub sample, while further sub samples are retained "blind" in order for subsequent use in confirming and validating the initial analysis[14]. The basic idea is to set aside some of the data randomly for training a model, then the data remained will be used to test the performance of the model. In this paper, a ten-folder cross-validation is used to test MCQP's performance as shown in Algorithm 2. The data gathered is divided into two groups. The training group is composed of 20 ST records and 20 healthy records and the testing group is composed of $20 \mathrm{ST}$ records and 60 healthy records. The process to select training and testing sets is described as follows: first, 20 ST records and 20 healthy records are randomly selected from the dataset. Then, they are combined to form a single training dataset, with the remained 20 ST records and 60 healthy records merged into a testing set.

\section{Algorithm 2}

Input: The data set $A=\{A 1, A 2, \ldots, A n\}$, boundary $b$

Output: Training accuracy $R_{t r}$, testing accuracy $R_{t s}$

Begin

Repeat ten times

Step1. Generate the training set $\{\mathrm{TR}\}$ and testing set $\{\mathrm{TS}\}$.

Step2. Sort all of the 24 attributes of $X^{*}$, the larger $X^{*}$, the more important of this attribute.

Step3. Calculate the score of each record $A i$ and get score array Score $[\mathrm{i}]=A i X^{*}$, for $\forall A i \in A$.

Step4. Compare Score[i] with boundary $b$, if $A i \in G 1$ with Score[i] $<b$, or $A i \in G 2$ with Score $[\mathrm{i}] \geq b$, then increase the number of correctly classified records $N_{t r}$ in training set and $N$ ts in testing set.

Step5. Calculate the classification performance both on training set ( $N_{t r} /|T R|$ ) and testing set ( $N_{t s} /|T S|$ ) and return the training accuracy Rtr and testing accuracy $R t s$.

End.

\subsection{Experiment Results}

Table 2 shows the ten-folder cross-validation result on the financial ratios dataset. The columns "ST" and "H" refer to the number of records that are correctly classified as 
"ST" and "Healthy", respectively. The column "Accuracy" is calculated using correctly classified records divided by the total records in that class.

From Table 2, we can see that the average accuracies of 10 groups training sets is $98.00 \%$ on the ST companies and $100 \%$ on the healthy companies, while the average accuracies of 10 groups testing sets is $90.00 \%$ on the ST companies and $96.50 \%$ on the healthy companies. The results indicate that a good separation of the ST class and Healthy class is observed with MCQP model.

Table 2. Results of MCQP model on financial ratios dataset

\begin{tabular}{|c|c|c|c|c|c|c|c|c|}
\hline \multirow{2}{*}{$\begin{array}{l}\text { Cross- } \\
\text { validation }\end{array}$} & \multicolumn{4}{|c|}{ Training Set } & \multicolumn{4}{|c|}{ Testing Set } \\
\hline & ST & Accuracy & $\mathrm{H}$ & Accuracy & ST & Accuracy & $\mathrm{H}$ & Accuracy \\
\hline DataSet 1 & 20 & $100.00 \%$ & 20 & $100.00 \%$ & 16 & $80.00 \%$ & 59 & $98.33 \%$ \\
\hline DataSet 2 & 20 & $100.00 \%$ & 20 & $100.00 \%$ & 18 & $90.00 \%$ & 56 & $93.33 \%$ \\
\hline DataSet 3 & 19 & $95.00 \%$ & 20 & $100.00 \%$ & 18 & $90.00 \%$ & 52 & $86.67 \%$ \\
\hline DataSet 4 & 19 & $95.00 \%$ & 20 & $100.00 \%$ & 20 & $100.00 \%$ & 57 & $95.00 \%$ \\
\hline DataSet 5 & 20 & $100.00 \%$ & 20 & $100.00 \%$ & 17 & $85.00 \%$ & 60 & $100.00 \%$ \\
\hline DataSet 6 & 20 & $100.00 \%$ & 20 & $100.00 \%$ & 17 & $85.00 \%$ & 60 & $100.00 \%$ \\
\hline DataSet 7 & 20 & $100.00 \%$ & 20 & $100.00 \%$ & 18 & $90.00 \%$ & 59 & $98.33 \%$ \\
\hline DataSet 8 & 19 & $90.00 \%$ & 20 & $100.00 \%$ & 19 & $95.00 \%$ & 57 & $95.00 \%$ \\
\hline DataSet 9 & 20 & $100.00 \%$ & 20 & $100.00 \%$ & 19 & $95.00 \%$ & 60 & $100.00 \%$ \\
\hline DataSet 10 & 20 & $100.00 \%$ & 20 & $100.00 \%$ & 18 & $90.00 \%$ & 59 & $98.33 \%$ \\
\hline Average & & $98.00 \%$ & & $100.00 \%$ & & $90.00 \%$ & & $96.50 \%$ \\
\hline
\end{tabular}

\subsection{Comparison of MCQP with Logistic Regression and SVM}

Table 3 exhibits the comparison results of MCQP, Logistic Regression and SVM. The first column lists the three algorithms. The second column is the recall rate of ST companies (more attention are paid to capture ST companies). And the third column is the accuracy of correctly classified companies (including both ST and healthy companies). It was found that the logistic regression model is slightly better than MCQP model on accuracy. However, MCQP model performs a little better on recall rate. Actually, recall rate is more important than accuracy as the misclassification of a real ST company will lead to a heavy loss. SVM achieves the least recall rate and accuracy. The reason may be that when doing classification, SVM chooses some marginal points as the delegates in each class, and it also thinks maximizing the distance of the delegates is maximizing the distance of each class. However, since our financial ratios dataset of the listed manufacturing companies probably obeys the Gaussian distribution, which means the points around the center is of the most important, choosing the marginal points as the delegates is not persuasive.

Table 3. Comparison of MCQP, Logistic, SVM

\begin{tabular}{lcc}
\hline \multirow{2}{*}{ Algorithms } & \multicolumn{2}{c}{ Testing records } \\
\cline { 2 - 3 } & recall & Accuracy \\
\hline MCQP & $90.00 \%$ & $94.88 \%$ \\
Logistic Regression & $87.50 \%$ & $95.00 \%$ \\
SVM & $82.50 \%$ & $92.50 \%$ \\
\hline
\end{tabular}




\section{Conclusion}

How to effectively predict financial distress becomes an urgent need for bank loan officers, investors, creditors, regulators and auditors. As a promising data mining approach, Multiple Criteria Quadratic Programming (MCQP) method has been extensively applied in many business activities. In this paper, we introduced MCQP into predicting financial distress of China' listed manufacturing companies. At the beginning, we collected and built a real-life listed manufacturing companies' dataset, which has 120 records with 24 attributes. Then we tested MCQP on this dataset using ten-folder cross-validation. Finally, we compared MCQP with Logistic Regression and SVM. The experimental results show that MCQP is accurate and stable for predicting the financial distress, and moreover, MCQP is superior to Logistic Regression and SVM models in the sense of accuracy and average recall rate. Consequently, we can say that MCQP model is capable of predicting the financial distress of the listed manufacturing companies accurately and stably.

\section{Acknowledgement}

This research has been partially supported by a grant from National Natural Science Foundation of China (\#70621001, \#70531040, \#70501030, \#10601064, \#70472074), National Natural Science Foundation of Beijing \#9073020, 973 Project \#2004CB720103, Ministry of Science and Technology, China and BHP Billiton Co., Australia.

\section{References}

1. Ko, P.C., Lin, P.C.: An evolution-based approach with modularized evaluations to forecast financial distress. Knowledge based systems 19, 84-91 (2006)

2. Beaver, W.H.: Financial ratios as predictors of failure. Journal of Accounting Research 4, 71-111 (1966)

3. Altman, E.I.: Financial ratios, discriminant analysis and the prediction of corporate bankruptcy. Journal of Finance 23, 71-111 (1966)

4. Ohlson, J.A.: Financial ratios and the probabilistic prediction of bankruptcy. Journal of Accounting Research 18, 109-131 (1980)

5. Mensah, Y.M.: An examination of the stationarity of multivariate bankruptcy prediction models: A methodologi-cal study. Journal of Accounting Research 22, 380-395 (1984)

6. Casey, C., Bartczak, N.: Using operating cash flow data to predict financial distress: some extensions. Journal of Accounting Research 23, 384-401 (1985)

7. Gentry, J.A., Newbold, P., Whitford, D.T.: Classifying bankrupt firms with funds flow components. Journal of Accounting Research 23, 146-160 (1985)

8. Coats, P.K., Fant, L.F.: Recognizing financial distress patterns using a neural network tool. Financial Management 22, 142-155 (1993)

9. Zhang, G.P., Hu, M.Y., Patuwo, B.E., Indro, D.C.: Artificial neural networks in bankruptcy prediction: General framework and cross-validation analysis. European Journal of Operational Research 116, 16-32 (1999)

10. Hu, Y.-C., Ansell, J.: Measuring retail company performance using credit scoring techniques. European Journal of Operational Research 183, 1595-1606 (2007) 
11. Olson, D., Shi, Y.: Introduction to Business Data Mining. McGraw-Hill/Irwin (2007)

12. Fisher, R.A.: The Use of Multiple Measurements in Taxonomic Problems. Annals of Eugenics 7, 179-180 (1936)

13. An, L.T.H., Tao, P.D.: Solving a class of linearly constrained indefinite quadratic problem by D. C. algorithms. Journal of Global Optimization 11, 253-285 (1997)

14. Schneider, J.: Cross Validation,

http://www.cs.cmu.edu/schneide/tut5/node42.html 